\title{
Sept étapes à suivre pour des évaluations efficaces d'un conseil d'administration et des administrateurs
}

\author{
Geoffrey Kiel, Ph. D., Université of Notre Dame, Australie \\ James Beck, directeur général, Effective Governance Pty Ltd \\ Jacques Grisé, Ph. D., F. Adm. A., Université Laval ${ }^{1}$
}

\section{INTRODUCTION}

\section{Étant donné que le conseil d'administration est le chef décisionnel ultime de la société, il est responsable des actions et du rendement de la société.}

Lorsqu'une crise se produit au sein d'une société, comme celles qu'ont connues Nortel et Hollinger International, les intervenants, les médias, les organismes de réglementation et la collectivité se tournent vers le conseil d'administration pour trouver des réponses. Étant donné que le conseil d'administration est le chef décisionnel ultime de la société, il est responsable des actions et du rendement de la société. Le défi actuel que doivent relever les conseils d'administration consiste à accroître la valeur des organisations qu'ils gouvernent. Grâce à l'évaluation du rendement, les conseils d'administration peuvent s'assurer qu'ils ont les connaissances, les compétences et la capacité de relever ce défi. Plusieurs guides et normes de pratiques exemplaires reconnaissent cette notion. Ainsi, la Commission des valeurs mobilières de l'Ontario indique dans les lignes directrices sur la gouvernance des sociétés (NP 58-201) que le conseil d'administration, ses comités et chacun de ses administrateurs devraient faire l'objet régulièrement d'une évaluation à l'égard de leur efficacité et de leur contribution ${ }^{2}$.

Une publication du Collège des administrateurs de sociétés $(\mathrm{CAS})^{3}$, sous forme de questions et réponses sur la gouvernance, a été conçue à l'intention des administrateurs nommés par le gouvernement du Québec comme membre d'un conseil d'administration d'une société d'État ou d'un organisme gouvernemental. Celle-ci vise à répondre aux questions les plus courantes qu'un administrateur nouvellement nommé peut légitimement se poser en matière de gouvernance. On y indique qu' "une évaluation faite à intervalles périodiques est essentielle pour assurer le maintien d'une gouvernance efficace ». C'est dans cette perspective que cet article offrira une approche pratique en matière d'évaluations efficaces des conseils d'administration et des administrateurs. En effet, il suggère un cadre comportant sept étapes qui pose les questions clés que tous les conseils d'administration devraient prendre en considération lorsqu'ils planifient une évaluation.

\section{1. ÉVALUATION DES CONSEILS D'ADMINISTRATION}

L'évaluation du conseil d'administration est trop souvent perçue comme un mal nécessaire - un processus mécanique consistant à cocher des points sur une liste qui, en bout de ligne, a peu de valeur réelle pour le conseil d'administration si ce n'est pour satisfaire aux exigences en matière de conformité. Toutefois, un processus efficace d'évaluation du conseil d'administration peut donner lieu à une transformation. Même les conseils d'administration efficaces peuvent tirer profit d'une évaluation bien menée. Comme nous l'avons résumé dans le tableau 1, une évaluation menée adéquatement peut contribuer considérablement à des améliorations du rendement à trois niveaux: organisation, conseil d'administration et administrateur. Certains auteurs ${ }^{5}$, notent que « les conseils qui évaluent leurs membres et qui s'évaluent ont tendance à être plus efficaces que ceux qui ne le font pas ». Toutefois, il faut souligner que ces avantages ne sont possibles qu'au moyen d'une évaluation du conseil d'administration menée de manière appropriée. Si l'évaluation n'est pas faite correctement, cela peut causer de la méfiance parmi les membres du conseil d'administration et entre le conseil lui-même et la direction. 


\section{Tableau 1 : Avantages possibles d'une évaluation du conseil d'administration}

\begin{tabular}{|c|c|c|c|}
\hline Avantages & Pour l'organisation & $\begin{array}{l}\text { Pour le conseil } \\
\text { d'administration }\end{array}$ & Pour les administrateurs \\
\hline Leadership & $\begin{array}{l}\text { - Donne le ton en matière de } \\
\text { rendement et détermine la } \\
\text { culture de l'organisation } \\
\text { - Sert de modèle pour le PDG et } \\
\text { l'équipe de la haute direction }\end{array}$ & $\begin{array}{l}\text { - Un président efficace utilisant } \\
\text { l'évaluation du conseil } \\
\text { d'administration fait preuve de } \\
\text { leadership pour les autres } \\
\text { membres du conseil } \\
\text { - Démontre la vision à long } \\
\text { terme du conseil } \\
\text { d'administration } \\
\text { - Comportements de leadership } \\
\text { approuvés et encouragés }\end{array}$ & $\begin{array}{l}\text { - Démontre l'engagement à } \\
\text { l'égard d'améliorations au } \\
\text { plan individuel }\end{array}$ \\
\hline $\begin{array}{l}\text { Clarification du } \\
\text { rôle }\end{array}$ & $\begin{array}{l}\text { - Permet d'établir clairement } \\
\text { une distinction entre les rôles } \\
\text { du PDG, de la direction et du } \\
\text { conseil d'administration } \\
\text { - Facilite les principes de } \\
\text { délégation appropriés }\end{array}$ & $\begin{array}{l}\text { - Clarifie le rôle de } \\
\text { l'administrateur et du comité } \\
\text { - Établit une norme du conseil } \\
\text { d'administration pour les rôles }\end{array}$ & $\begin{array}{l}\text { - Clarifie les fonctions de } \\
\text { chaque administrateur } \\
\text { - Clarifie les attentes }\end{array}$ \\
\hline Travail en équipe & $\begin{array}{l}\text { Renforce les relations avec le } \\
\text { conseil d'administration, le } \\
\text { PDG et la direction }\end{array}$ & $\begin{array}{l}\text { - Permet de bâtir la confiance } \\
\text { entre les membres du conseil } \\
\text { d'administration } \\
\text { - Favorise une participation } \\
\text { active } \\
\text { - Favorise un engagement et un } \\
\text { sentiment d'appartenance }\end{array}$ & $\begin{array}{l}\text { - Favorise la participation de } \\
\text { chaque administrateur } \\
\text { - Favorise un engagement et un } \\
\text { sentiment d'appartenance } \\
\text { - Clarifie les attentes }\end{array}$ \\
\hline $\begin{array}{l}\text { Reddition de } \\
\text { comptes }\end{array}$ & $\begin{array}{l}\text { - Améliore les rapports avec les } \\
\text { intervenants (par exemple, les } \\
\text { investisseurs, les marchés } \\
\text { financiers) } \\
\text { - Améliore les normes de } \\
\text { gouvernance de la société } \\
\text { - Clarifie les délégations }\end{array}$ & $\begin{array}{l}\text { - Fait en sorte que le conseil } \\
\text { d'administration met l'accent } \\
\text { sur les obligations à l'égard } \\
\text { des intervenants } \\
\text { - Permet de s'assurer que le } \\
\text { conseil d'administration est } \\
\text { une organisation assurant une } \\
\text { surveillance adéquate }\end{array}$ & $\begin{array}{l}\text { - Permet de s'assurer que les } \\
\text { administrateurs comprennent } \\
\text { leurs fonctions et leurs } \\
\text { responsabilités juridiques } \\
\text { - Établit les attentes en matière } \\
\text { de rendement pour chaque } \\
\text { membre du conseil } \\
\text { d'administration }\end{array}$ \\
\hline Prise de décisions & $\begin{array}{l}\text { - Clarifie la vision stratégique et } \\
\text { les buts de la société } \\
\text { - Améliore la prise de décisions } \\
\text { de l'organisation }\end{array}$ & $\begin{array}{l}\text { - Clarifie la vision stratégique } \\
\text { - Facilite la détermination des } \\
\text { lacunes en matière de } \\
\text { compétences du conseil } \\
\text { d'administration } \\
\text { - Améliore la capacité du } \\
\text { conseil d'administration à } \\
\text { prendre des décisions }\end{array}$ & $\begin{array}{l}\text { - Permet de déterminer les } \\
\text { secteurs où l'administrateur } \\
\text { doit améliorer ses } \\
\text { compétences } \\
\text { - Permet de déterminer les } \\
\text { secteurs où les compétences } \\
\text { de l'administrateur peuvent } \\
\text { être utilisées à meilleur escient }\end{array}$ \\
\hline Communication & $\begin{array}{l}\text { - Améliore les rapports avec les } \\
\text { intervenants } \\
\text { - Améliore les rapports entre le } \\
\text { conseil d'administration et la } \\
\text { direction } \\
\text { - Améliore les rapports entre le } \\
\text { conseil d'administration et le } \\
\text { PDG }\end{array}$ & $\begin{array}{l}\text { - Améliore les rapports entre le } \\
\text { conseil d'administration et la } \\
\text { direction } \\
\text { - Permet de bâtir la confiance } \\
\text { entre les membres du conseil } \\
\text { d'administration }\end{array}$ & $\begin{array}{l}\text { - Renforce les relations } \\
\text { personnelles entre les } \\
\text { administrateurs }\end{array}$ \\
\hline $\begin{array}{l}\text { Activités du conseil } \\
\text { d'administration }\end{array}$ & $\begin{array}{l}\text { - Permet de s'assurer qu'il } \\
\text { existe un cadre stratégique de } \\
\text { haut niveau approprié pour } \\
\text { orienter l'organisation }\end{array}$ & $\begin{array}{l}\text { - Réunions plus efficaces } \\
\text { - Meilleure gestion du temps }\end{array}$ & $\begin{array}{l}\text { - Permet d'épargner du temps } \\
\text { aux administrateurs } \\
\text { - Accroît l'efficacité de chaque } \\
\text { participant }\end{array}$ \\
\hline
\end{tabular}




\section{VERS UN MODÈLE D'ÉVALUATION DES CONSEILS D'ADMINISTRATION}

Même si la gravité des problèmes de gouvernance et l'éventail des enjeux auxquels les conseils d'administration sont confrontés peuvent varier, il y a encore plusieurs décisions clés qui touchent tous les conseils d'administration pour ce qui est de la mise en œuvre d'un processus d'évaluation. Un cadre efficace repose sur les réponses données d'un commun accord par le conseil d'administration aux sept questions clés illustrées dans la figure 1 . Bien que ces questions doivent être posées pour toutes les évaluations du conseil d'administration, les réponses combinées peuvent différer passablement. Par conséquent, même si les questions sont communes pour chaque conseil, la portée, la complexité et le coût des évaluations peuvent varier considérablement.

Bien que le cadre ci-après soit présenté de manière séquentielle, en fait la plupart des conseils d'administration ne suivront pas un tel processus linéaire, certaines décisions seront prises en même temps. Ainsi, on pourra déterminer simultanément «les personnes qui seront évaluées » et «le responsable de l'évaluation». Toutefois, il faudra à un certain moment pour répondre à chacune de ces questions.

\section{Figure 1 : Cadre pour une évaluation du conseil d'administration ${ }^{7}$}

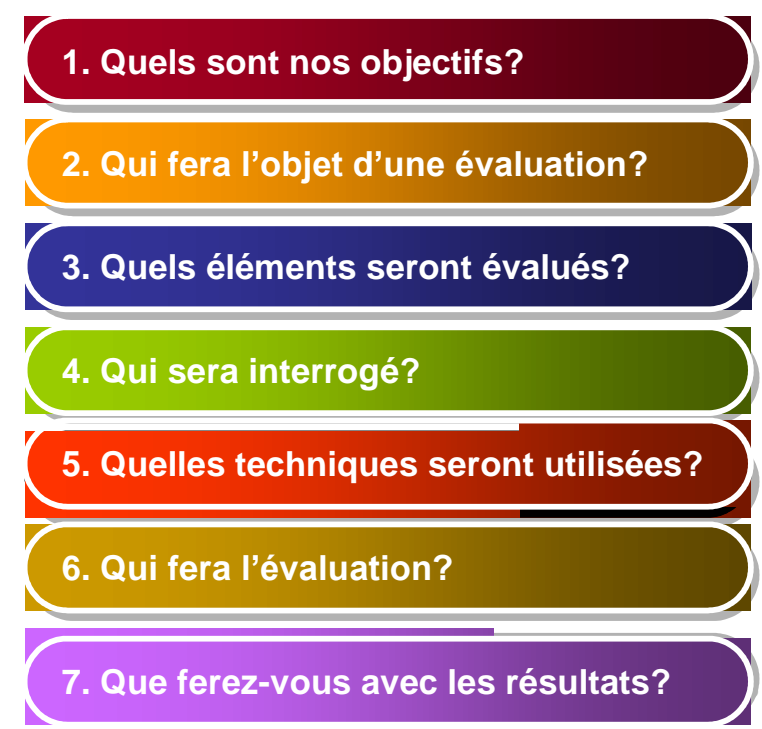

\section{PROCESSUS D'ÉVALUATION DES CONSEILS D'ADMINISTRATION}

\section{Étape 1}

Quels sont nos objectifs?

L'étape 1 consiste à établir les objectifs que le conseil d'administration essaie d'atteindre. Des objectifs clairement définis permettent au conseil d'administration de fixer des buts précis pour l'évaluation et de prendre des décisions quant à la portée de l'examen. Des questions telles que la complexité du problème de rendement, la taille du conseil d'administration, l'étape du cycle de vie de l'organisation et les développements importants dans l'environnement concurrentiel de l'entreprise détermineront les points que le conseil d'administration souhaite évaluer. De même, la gravité des problèmes auxquels le conseil d'administration fait face et la disponibilité de ressources suffisantes pour mener une évaluation détermineront la portée de l'examen (combien de personnes y participeront? combien de temps? combien d'argent fautil allouer?).

La plupart des conseils d'administration doivent d'abord déterminer le motif prépondérant du processus d'évaluation. En général, la réponse à 
cette question se trouve dans l'une des deux catégories suivantes :

- leadership corporatif - par exemple, «nous voulons démontrer clairement notre engagement à l'égard de la gestion du rendement »;

- la résolution de problèmes - par exemple, « nous ne semblons pas avoir les habiletés, les compétences ou la motivation appropriées au sein du conseil d'administration ».

\section{Étape 2 \\ Qui fera l'objet d'une évaluation?}

\section{Une évaluation efficace exige que le conseil d'administration sélectionne les personnes ou les groupes les plus pertinents pour l'examen, selon ses objectifs.}

Des évaluations exhaustives de la gouvernance peuvent comporter l'examen du rendement d'un grand nombre de personnes et de groupes. Les conseils d'administration doivent prendre en considération trois groupes : le conseil dans son ensemble (y compris les comités); les administrateurs (y compris le rôle du président); le personnel clé responsable de la gouvernance. Cependant, des considérations comme les contraintes reliées au coût ou au facteur temps font souvent obstacle à un tel examen de vaste portée.

Un conseil d'administration peut avoir un objectif très précis pour le processus d'examen qui ne nécessite pas l'évaluation de toutes les personnes et de tous les groupes établis. Dans les deux cas, une évaluation efficace exige que le conseil d'administration sélectionne les personnes ou les groupes les plus pertinents pour l'examen, selon ses objectifs. Pour prendre cette décision, nous recommandons que la liste des participants éventuels à l'examen soit progressivement réduite à une sélection pragmatique des personnes faisant l'objet de l'évaluation.

Un enjeu commun lorsqu'il faut choisir les personnes à évaluer est de déterminer s'il faut se concentrer uniquement sur le conseil d'administration dans son ensemble ou inclure également l'évaluation de chacun de ses administrateurs.
L'évaluation courante du conseil d'administration dans son ensemble peut être perçue comme un processus permettant de s'assurer que les administrateurs acquièrent une compréhension commune de leur rôle et de leurs responsabilités en matière de gouvernance. Bien que l'évaluation du conseil dans son ensemble soit excellente comme outil de familiarisation pour des conseils d'administration inexpérimentés, le désavantage est que l'évaluation des groupes peut donner seulement une indication limitée des problèmes de rendement et de gouvernance. Par conséquent, certains conseils d'administration optent pour l'évaluation des comités du conseil d'administration, de chacun de ses administrateurs et du président pour mieux connaître son fonctionnement.

L'évaluation courante du conseil d'administration dans son ensemble peut être perçue comme un processus permettant de s'assurer que les administrateurs acquièrent une compréhension commune de leur rôle et de leurs responsabilités en matière de gouvernance.

Pour avoir une idée objective du rendement de chaque administrateur, une évaluation par les pairs est souhaitable, car les membres du conseil s'évaluent entre eux. Il est donc possible d'avoir un aperçu plus global des forces et des faiblesses de chaque administrateur et de leur contribution à l'efficacité du conseil d'administration. L'évaluation peut également servir à cerner les lacunes en matière de compétences au sein du conseil d'administration ou les problèmes de communication entre les administrateurs. Si une évaluation est menée pour chaque administrateur, il est primordial que les résultats de cet examen présentent une corrélation avec les résultats de l'évaluation du conseil dans son ensemble pour valider la pertinence des recommandations.

Pour avoir une idée objective du rendement de chaque administrateur, une évaluation par les pairs est souhaitable, car les membres du conseil s'évaluent entre eux. 
Étape 3

Quels éléments seront évalués?

Après avoir établi les objectifs de l'évaluation et les personnes et les groupes qui seront évalués, il faut définir ces objectifs en plusieurs thèmes précis afin de s'assurer que l'évaluation: clarifie les problèmes éventuels, détermine les causes premières de ces problèmes et met, dans la mesure du possible, à l'essai l'aspect pratique de certaines solutions en matière de gouvernance.

Cette étape est nécessaire peu importe si le conseil souhaite des améliorations générales ou particulières du rendement. La détermination et la précision des éléments à évaluer seront utiles pour les conseils d'administration cherchant à améliorer des domaines aussi variés que les procédures $\mathrm{du}$ conseil d'administration, les habiletés, les compétences et la motivation des administrateurs ou même les rapports à la salle du conseil.
La détermination et la précision des éléments à évaluer seront utiles pour les conseils d'administration cherchant à améliorer des domaines aussi variés que les procédures du conseil d'administration, les habiletés, les compétences et la motivation des administrateurs ou même les rapports à la salle du conseil.

Nous suggérons que les conseils d'administration envisagent leurs objectifs précis à la lumière d'un cadre de gouvernance prédominant pour établir les rôles que le conseil souhaite remplir sur certains domaines, illustrés au tableau 2.

Naturellement, la liste exhaustive des domaines d'analyse devra être conciliée avec la portée de l'évaluation et les ressources disponibles pour le projet. À cette étape, il est possible de mener une évaluation réaliste des ressources disponibles dont un élément est la disponibilité des administrateurs et d'autres employés clés responsables de la gouvernance.

Tableau 2 : Rôles génériques du conseil d'administration ${ }^{8}$

\begin{tabular}{|ll|}
\hline & Rôle du conseil d'administration \\
\hline 1. & Stratégie \\
\hline 2. & PDG \\
\hline 3. & Surveillance \\
\hline 4. & Aperçu de la gestion des risques \\
\hline 5. & Aperçu de la conformité \\
\hline 6. & Cadre stratégique \\
\hline 7. & Établissement de réseaux \\
\hline 8. & Communication avec les intervenants \\
\hline 9. & Prise de décisions \\
\hline 10. & Gouvernance efficace \\
\hline
\end{tabular}




\section{Étape 4 \\ Qui sera interrogé?}

La vaste majorité des évaluations du conseil d'administration et des administrateurs se concentrent exclusivement sur le conseil d'administration (et peut-être le PDG) en tant qu'unique source d'information pour le processus d'évaluation. Toutefois, cette approche ne tient pas compte d'autres sources d'information qui peuvent être précieuses. Les participants à l'évaluation peuvent se trouver au sein ou à l'extérieur de la société. À l'interne, les membres du conseil d'administration, le PDG, les cadres supérieurs et, dans certains cas, d'autres gestionnaires et employés peuvent avoir l'information nécessaire pour transmettre des commentaires au sujet de certains éléments du système de gouvernance d'une société. À l'externe, les propriétaires et les membres et même les marchés financiers peuvent fournir des données utiles pour l'examen. De même, dans certaines situations, des ministères fédéraux, des clients importants et des fournisseurs peuvent avoir des liens étroits avec le conseil d'administration et être en mesure de fournir des renseignements utiles sur son rendement.

Après avoir examiné toutes les sources possibles d'information ainsi que leurs avantages et leurs inconvénients relatifs, le responsable doit déterminer les sources qu'il faut inclure dans l'examen. Il faut à cette fin comprendre les trois points. D'abord, et à la lumière des questions spécifiques établies à l'étape précédente, qui a les connaissances nécessaires pour faire une évaluation valide et fiable. Ensuite, déterminer quel est le degré d'expérience du conseil d'administration, quelle est son ouverture à l'égard du processus d'évaluation et quelles sont les répercussions sur la détermination des personnes qu'il faut rencontrer. Enfin, comprendre quelles ressources sont disponibles pour recueillir l'information auprès des sources nécessaires.

\section{Étape 5}

Quelles techniques seront utilisées?

Selon le degré de formalité, les objectifs de l'évaluation et les ressources disponibles, les conseils d'administration peuvent choisir parmi diverses techniques qualitatives et quantitatives. Les données quantitatives se présentent sous forme de chiffres. Elles peuvent servir à poser des questions sur la quantité ou le nombre. Des questions telles que «quoi», «comment», «pourquoi », «quand » et « où » utilisent des méthodes de recherche qualitative.

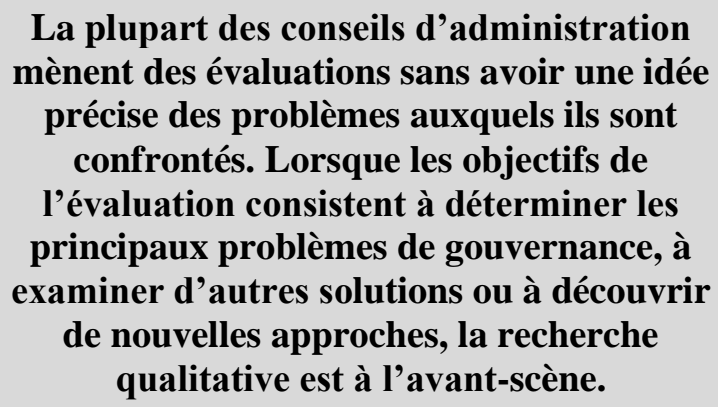

La plupart des conseils d'administration mènent des évaluations sans avoir une idée précise des problèmes auxquels ils sont confrontés. Lorsque les objectifs de l'évaluation consistent à déterminer les principaux problèmes de gouvernance, à examiner d'autres solutions ou à découvrir de nouvelles approches, la recherche qualitative est à l'avant-scène. Toutefois, les données qualitatives comportent plusieurs inconvénients.

Le principal inconvénient est que l'interprétation des résultats exige du jugement de la part de la personne menant l'examen et l'analyse. Il est donc mieux indiqué de recourir à des chercheurs expérimentés pour cette tâche et de faire en sorte que plusieurs participants examinent les conclusions pour empêcher la subjectivité. On peut également atténuer la partialité en utilisant des techniques à la fois quantitatives et qualitatives.

Les trois principales méthodes utilisées pour recueillir des données qualitatives dans les évaluations de la gouvernance sont les entrevues, l'observation du conseil d'administration et l'analyse des documents. Premièrement, l'entrevue offre l'occasion unique de recueillir des données complexes et précieuses. C'est une excellente façon d'évaluer les perceptions des administrateurs, le sens et la construction de la 
réalité en demandant de l'information de manière à leur permettre de s'exprimer dans leurs propres termes. Deuxièmement, l'observation d'une réunion du conseil d'administration est tout particulièrement utile lorsque les objectifs de l'évaluation ont trait aux questions de la dynamique ou des rapports entre les personnes. Troisièmement, les documents peuvent également représenter une source précieuse d'information pour le processus d'évaluation de la gouvernance. Il peut s'agir d'une méthode de triangulation à utiliser de concert avec d'autres techniques de collecte de données.

Bien que les données quantitatives ne soient pas aussi riches en information que les données qualitatives, elles ont l'avantage d'être précises et facilement mesurables.

Bien que les données quantitatives ne soient pas aussi riches en information que les données qualitatives, elles ont l'avantage d'être précises et facilement mesurables. Les sondages sont de loin la forme la plus courante de technique quantitative utilisée pour les évaluations de la gouvernance et ils peuvent être un outil important de collecte de l'information. Il est toutefois essentiel de comprendre que les sondages sont des instruments permettant de mesurer les attitudes. Il n'y a pas de méthodologie idéale. Les techniques de recherche doivent être adaptées selon les objectifs de l'évaluation et le contexte du conseil d'administration.

\section{Étape 6}

Qui fera l'évaluation?

\section{Dans le cas des évaluations externes, des experts-conseils ou d'autres conseillers généraux ayant une expertise dans les domaines de la gouvernance d'une société et de l'évaluation du rendement pourraient diriger le processus.}

À cette étape, il faut déterminer la personne la plus compétente pour mener l'évaluation. S'il s'agit d'un examen interne, le président peut mener l'évaluation.
Toutefois, pour des raisons d'impartialité, il peut être parfois plus approprié de déléguer l'évaluation à un administrateur non exécutif ou à un administrateur principal ou bien à un comité du conseil d'administration. Selon les étapes précédentes et les décisions prises à l'étape 7 quant à l'auditoire auquel les résultats seront transmis, les conseils d'administration bien établis envisagent plus souvent de participer à des évaluations externes pour assurer un degré d'indépendance et d'expertises en vue d'améliorer de manière proactive la gouvernance globale et la dynamique du conseil d'administration.

Dans le cas des évaluations externes, des experts-conseils ou d'autres conseillers généraux ayant une expertise dans les domaines de la gouvernance d'une société et de l'évaluation du rendement pourraient diriger le processus. Cependant, en raison de sa nature spécialisée, l'examen du conseil d'administration nécessite souvent des compétences qui ne font pas partie habituellement des habiletés de nombreux conseillers généraux. De même, un expertconseil recruté tout spécialement pour mener l'évaluation peut être jugé plus indépendant qu'un examinateur qui a un lien avec l'entreprise (tel qu'un avocat général ou un vérificateur). Par ailleurs, les experts-conseils auront un large éventail de connaissances des différentes pratiques à la salle du conseil et des analyses comparative du rendement.

Un compromis entre les deux approches est d'utiliser une évaluation en ligne du conseil d'administration pour préparer de manière confidentielle un rapport d'évaluation qui peut être produit par le président ou l'administrateur principal.

\section{Étape 7}

\section{Que ferez-vous avec les résultats?}

Les objectifs de l'examen devraient être le facteur déterminant lors du choix des personnes auxquelles les résultats seront transmis. La plupart du temps, le conseil d'administration vise principalement à convenir d'une série de mesures qu'il peut prendre pour améliorer la gouvernance. Puisque l'efficacité du système de gouvernance d'une organisation repose sur les employés au sein de l'entreprise, il est essentiel 
de transmettre les résultats aux intervenants internes pour les conseils d'administration souhaitant améliorer le rendement. Étant donné que presque tous les examens de la gouvernance sont menés en vue d'améliorer le système de gouvernance, les conseils d'administration doivent rarement décider s'ils transmettent les résultats à l'interne. Ils doivent plutôt déterminer les personnes qui doivent connaître les résultats au sein de l'organisation.

Lorsque les résultats de l'évaluation portent sur le rendement de chaque administrateur, l'approche généralement acceptée consiste à ce que le président du conseil d'administration ou le responsable discute des résultats avec chacun d'entre eux. Les administrateurs peuvent être tenus de discuter de leurs propres résultats autour de la table du conseil, un processus qui peut mener à une bien meilleure compréhension mutuelle.

Étant donné que le conseil d'administration dans son ensemble est responsable de son rendement, les résultats de l'examen lui seront transmis dans toutes les circonstances, sauf dans des circonstances exceptionnelles. Lorsque les objectifs de l'évaluation sont entièrement axés sur le conseil d'administration, ses membres discuteront simplement des résultats entre eux. Habituellement, le conseil d'administration, le PDG et le secrétaire général examineront les résultats autour de la table de la salle du conseil. Il ne sera peut-être pas nécessaire de communiquer les résultats à d'autres personnes. Lorsque les résultats de l'évaluation portent sur le rendement de chaque

\section{CONCLUSION}

\section{L'évaluation du rendement des conseils}

d'administration peut être un processus continu et non pas une activité annuelle. Les conseils d'administration hautement performants ont tendance à concevoir d'autres mécanismes qu'un examen annuel pour assurer une amélioration continue du rendement.

À part les sept questions clés d'une évaluation, les conseils d'administration doivent déterminer la fréquence des évaluations de leur rendement. administrateur, l'approche généralement acceptée consiste à ce que le président du conseil d'administration ou le responsable discute des résultats avec chacun d'entre eux. Les administrateurs peuvent être tenus de discuter de leurs propres résultats autour de la table du conseil, un processus qui peut mener à une bien meilleure compréhension mutuelle.

Lorsque l'évaluation du conseil d'administration a pour objectif d'évaluer la qualité des rapports entre le conseil d'administration et la direction, les résultats de l'évaluation seront généralement transmis à l'équipe de la haute direction. Certaines organisations choisissent de transmettre un résumé des résultats de l'évaluation du conseil d'administration sur une grande échelle au sein de l'organisation.

Dans certaines circonstances, le conseil d'administration aura pour objectif de bâtir sa réputation en matière de transparence ou d'établir des relations avec des intervenants externes. Le conseil d'administration devrait alors envisager de communiquer une partie ou la totalité des résultats de son examen à ces intervenants. La transmission des résultats de l'évaluation démontre que le conseil d'administration prend la gouvernance au sérieux et qu'il est déterminé à améliorer son rendement. Il est évident qu'il faut établir un juste équilibre entre la transparence d'une part et la nécessité pour les propriétaires et les membres de maintenir leur confiance en la capacité et en l'efficacité du conseil d'administration, d'autre part.

L'examen annuel est la méthode d'évaluation la plus couramment recommandée. Toutefois, un événement annuel prévisible peut être dépassé et ne plus ajouter de valeur. Par conséquent, il est important d'essayer différents styles et différentes techniques d'évaluation pour que le processus demeure intéressant et s'assurer qu'il permet d'améliorer le rendement.

L'évaluation du rendement des conseils d'administration peut être un processus continu et non pas une activité annuelle. Les conseils d'admi- 
nistration hautement performants ont tendance à concevoir d'autres mécanismes qu'un examen annuel pour assurer une amélioration continue $\mathrm{du}$ rendement. Une option consiste à examiner l'efficacité de chaque réunion du conseil d'administration. Il s'agit d'une technique simple permettant de s'assurer que les questions relatives au rendement demeurent «au premier plan » pour le conseil d'administration. C'est un moyen facile de recueillir rapidement des commentaires et de promouvoir la discussion et l'interaction entre les membres du conseil d'administration et il exige peu de temps ou d'effort pour le mettre en place.

Les conseils d'administration doivent également reconnaître que le processus d'évaluation est une activité efficace qui favorise l'esprit d'équipe et qui permet de façonner l'éthique.

L'évaluation du rendement prend de plus en plus d'importance pour les conseils d'administration et les administrateurs. Elle comporte des avantages pour les administrateurs, les conseils d'administration et les sociétés pour lesquelles ils travaillent. Les conseils d'administration doivent également reconnaitre que le processus d'évaluation est une activité efficace qui favorise l'esprit d'équipe et qui permet de façonner l'éthique.

Une publication de l'École d'administration publique du Québec, produite en collaboration avec le Collège des administrateurs de sociétés, présente une section traitant de l'évaluation de la performance du conseil d'administration. Selon les auteurs, «L'évaluation est une composante essentielle d'une saine gouvernance d'entreprise. Elle permet de jeter un regard sur la façon dont les décisions ont été prises et sur la manière d'exercer la gestion des activités de l'organisation et ce, dans une perspective d'amélioration continue [...] Il incombe au président du conseil d'instaurer une culture d'évaluation du rendement et de la performance. Pour ce faire, il doit veiller à la mise en place d'un processus d'évaluation clair, à l'élaboration de règles et d'outils pertinents, à la définition des responsabilités de chaque intervenant dans le processus d'évaluation, à la diffusion de l'information et à la mise en place des correctifs nécessaires ». Dans cette publication, on présente dix questionnaires détaillés d'évaluations qui concernent diverses cibles telles que l'évaluation du conseil, l'évaluation du fonctionnement du conseil, l'évaluation du président du conseil, l'évaluation d'un membre du conseil, l'évaluation du comité de gouvernance et d'éthique, l'évaluation du comité de vérification, l'évaluation du comité des ressources humaines, l'évaluation du fonctionnement d'un comité, l'évaluation d'un membre du comité et l'évaluation du président d'un comité.

Notons enfin que les conseils d'administration négligent souvent le processus de mobilisation lorsqu'ils entreprennent des évaluations; malheureusement, les conseils d'administration qui ne font pas participer leurs membres ratent cette occasion unique pour élaborer une série commune de normes pour le conseil d'administration et inculquer une culture positive au sein du conseil et de l'organisation. En bref, le processus est aussi important que le contenu.

\section{BIBLIOGRAPHIE ET NOTES}

Il est possible de communiquer avec le professeur Geoffrey Kiel et James Beck par courriel à advisors@effectivegovernance.com.au ou James.Beck@effectivegovernance.com.au respectivement ou en se rendant sur le site www.effectivegovernance.com.au. Il est possible de communiquer avec le professeur Grisé par courriel à Jacques.grise@cas.ulaval.ca ou en se rendant sur le site Web du CAS :

${ }^{2}$ CVMO, Corporate Governance Guidelines (NP 58-201), section 3.18.

${ }^{3}$ Collège des administrateurs de sociétés, (2007). Être un administrateur de sociétés d'état: 16 questions et réponses sur la gouvernance, Faculté des sciences de l'administration, Université Laval, p. 22.

${ }^{4}$ Nadler, D A, Behan, B A, and Nadler, M B, (2006). Building Better Boards: A Blueprint for Effective Governance. San Francisco, Jossey-Bass, p. 230.

${ }^{5}$ Lawler III, E. E., Finegold, D. L., The Changing Face of Corporate Boards, Sloan Management Review, hiver 2005, p. 70.

${ }^{6}$ Kiel, G C, Nicholson, G J, and Barclay, M. A., (2005). Board, Director and CEO Evaluation, McGraw-Hill, Sydney, 13-14.

${ }^{7}$ Kiel, Nicholson, and Barclay, (2005). Board, Director and CEO Evaluation, p. 17.

${ }^{8}$ Pour plus de précisions au sujet de ces rôles, consultez le site www.effectivegovernance.com.au et sélectionnez «Services/Board Evaluation».

${ }^{9} \mathrm{ENAP}$, (2007). Les devoirs et responsabilités d'un conseil d'administration, Guide de référence, Bibliothèque et Archives nationales du Québec, 37-45. 


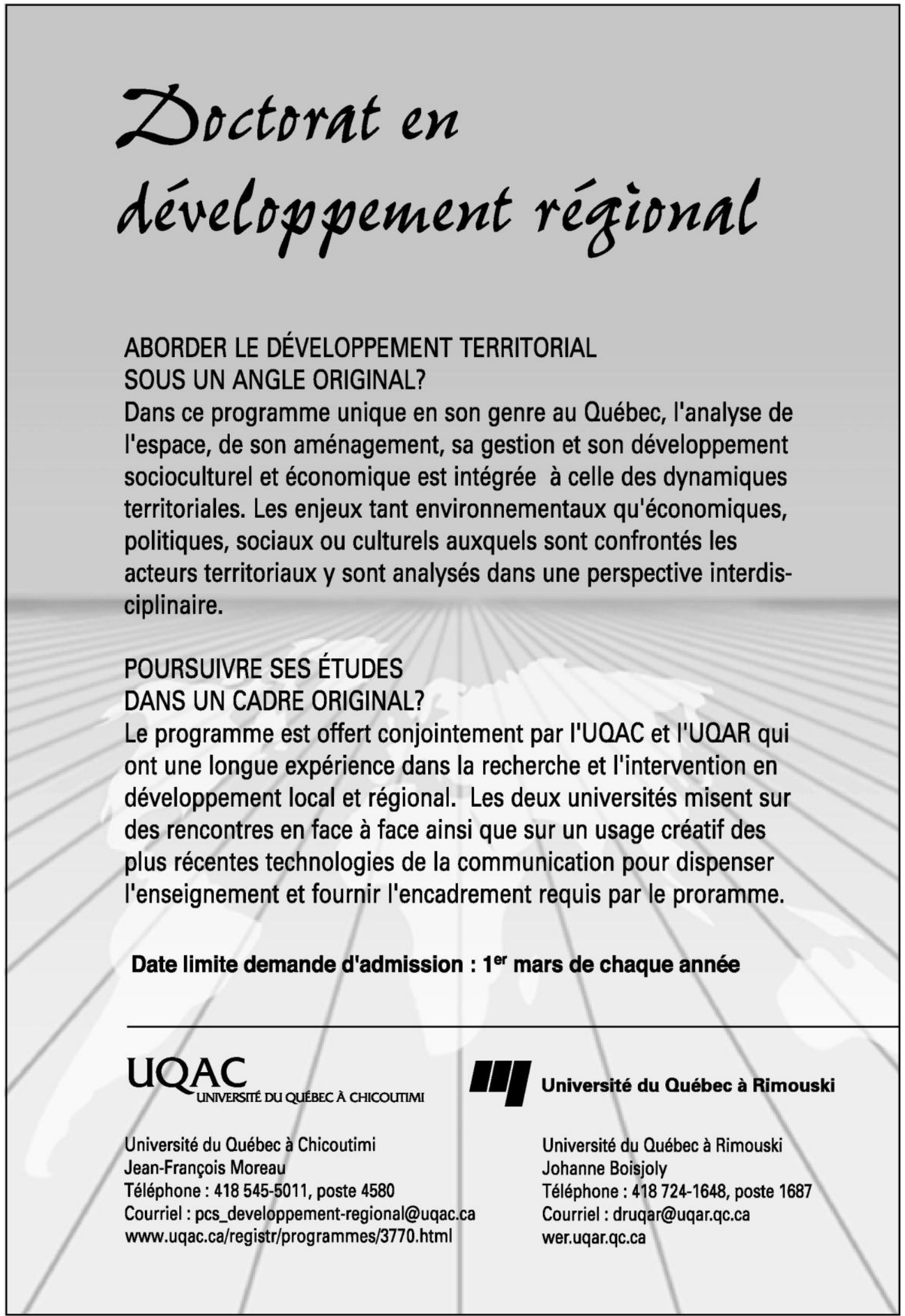

